\title{
Économie et langue : le cas occitan
}

The economy and language: the case of Occitan

\section{Alain Alcouffe}

\section{OpenEdition}

\section{Journals}

Édition électronique

URL : https://journals.openedition.org/lbl/1096

DOl : $10.4000 / \mathrm{lbl} .1096$

ISSN : 2727-9383

\section{Éditeur}

Université de Bretagne Occidentale - UBO

\section{Édition imprimée}

Date de publication : 1 juin 2015

Pagination : 239-259

ISBN : 979-10-92331-16-5

ISSN : 1270-2412

\section{Référence électronique}

Alain Alcouffe, «Économie et langue : le cas occitan», La Bretagne Linguistique [En ligne], 19 | 2015, mis en ligne le 01 mai 2021, consulté le 22 mai 2021. URL : http://journals.openedition.org//bl/1096 DOl : https://doi.org/10.4000/lbl.1096

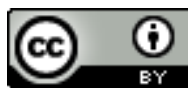

La Bretagne Linguistique est mise à disposition selon les termes de la Licence Creative Commons Attribution 4.0 International. 
Alain ALCOUFFE*

\section{Économie et langue : le cas occitan}

\section{Misère de l'économie et déclin linguistique}

$\mathrm{D}$

epuis 1229, à de multiples occasions, la défense de l'occitan a souvent établi un lien entre la situation de la langue et la situation économique et politique des régions occitanes. La redécouverte de la langue occitane au début du XIX ${ }^{\mathrm{e}}$ siècle par de Rochegude et Raynouard puis celle de la croisade des Albigeois par Napoléon Peyrat a coïncidé avec un retard du développement économique et en particulier industriel dont les causes sont multiples, tenant à la fois à une pauvreté relative en ressources naturelles (pauvreté en houille et fer) qu'à des voies de communication très insuffisantes. Très vite le décrochage économique a été attribué à un délaissement par un pouvoir central dont on connaît la lutte qu'il mène contre la langue occitane. On peut trouver une expression de cette mise en relation de l'économique et du politique dans les prises de position d'Urbain Vitry (1802-1863), l'architecte en chef de la ville de Toulouse dans son compte rendu de l'exposition universelle de Londres de 1851 :

«Sous la dynastie de 1830, un vaste système de chemins de fer, de cet agent si puissant de prospérité et de civilisation, se réalise dans le Nord de la France. Le Midi se trouve encore déshérité ! Son tour n'arrivera que plus tard, comme pour les

* Professeur émérite, Université de Toulouse, président de l'Ofici per l'Occitan. 
canaux. Pendant ce temps, le Nord prend constamment le devant, obéissant en quelque sorte à la loi si connue du mouvement des graves : les espaces parcourus sont comme les carrés des temps ; tandis que les départements méridionaux attendent encore l'impulsion première qui doit les faire progresser à leur tour ! Ils croyaient naguère pouvoir obtenir enfin une justice tardive, mais une sorte de fatalité venant paralyser des intentions réparatrices, a fait évanouir cette lueur d'espoir. On dirait qu'une autre croisade des hommes du Nord, renouvelée de celle des Albigeois, s'acharne une nouvelle fois contre le Midi pour y étouffer toute nationalité, toute source de vie et de prospérité ${ }^{1}$. »

Deux ans après que ces lignes aient été écrites, sept jeunes poètes provençaux emmenés par Frédéric Mistral et Joseph Roumanille fondaient le Félibrige le 21 mai 1854 dans le Vaucluse, qui entendait restaurer la langue provençale. Mistral devait publier en 1859 Mirèio (Mireille) qui lui vaudra le prix Nobel en 1904. Huit ans plus tard, il publie Calendal, dans lequel apparaît encore la farouche volonté d'indépendance des Provençaux. Il ira même en 1876 dans un hommage à la renaissance catalane jusqu'à faire de la langue l'outil de la libération dans un vers célèbre ${ }^{2}$ :

Car, de mourre-bourdoun, Qu'un pople toumbe esclau, Se tèn sa lèngo, ten la clau Que di cadèno lou deliéuro...
Car même si, face contre terre, Un peuple tombe en esclavage, S'il garde sa langue, il garde la clé Qui de ses chaînes le délivre...

\section{La révolte des vignerons de 1907}

Le décrochage industriel à partir de l'arrivée du chemin de fer en 1853 s'est accompagné d'un développement de la monoculture viticole rendant particulièrement sensible aux fluctuations du marché du vin l'économie des départements méridionaux qui fournissent près de la moitié de la production française de vins. Or, au début

1. Urbain VITRY, «Le département de la Haute-Garonne à l'Exposition de Londres ", Mémoires de l'Académie des sciences inscriptions et belles-lettres de Toulouse, 1852, t. II, 4e série, p. 226-237.

2. Frédéric Mistral, Odo i Troubaire Catalan, Lis Isclo d'Or, Avignon, Roumanille, 1980 [1876], p. 164-177. 
des années 1900, la production de vins industriels, la concurrence espagnole et italienne et l'arrivée de vins d'Algérie provoque un engorgement du marché du vin et en 1907 éclate une crise viticole et les masses rurales exaspérées par l'inertie gouvernementale «prennent parti pour elles-mêmes, pour leur spécificité de mœurs, de situation et même de langue ${ }^{3} \gg$. Dans cette révolte elles manifestaient l'usage de leur langue comme le prouvent les pancartes villageoises et les couplets qui accompagnaient les manifestants sur les routes d'Occitanie ${ }^{4}$. Cette conjonction d'une résurgence occitane et d'une révolte populaire fut saisie par le maire de Narbonne, le docteur Ferroul, qui était un sympathisant du Félibrige et un grand lecteur de Mistral et dont les discours enflammés faisaient écho à la Chanson de la Croisade albigeoise. Mais les démarches faites pour solliciter l'appui voire la présence de Mistral lui-même auréolé de son prix Nobel de littérature de 1904, dans ces manifestations gigantesques, furent vaines, ce qui devait arracher à Pierre Devoluy, alors à la tête du Félibrige, dans une lettre adressée à Jules Ronjat, un aveu plein d'amertume : «Aujourd'hui, j'ai entendu Calendal renié par son père, et il me faudra longtemps pour m'en remettre, si jamais je m'en remets ${ }^{5} . »$

Au lieu d'un soutien ferme qui aurait associé défense de la langue et revendication économique, Mistral se contenta d'une brève carte du 31 mai 1907 dans laquelle il écrivait : «Vive la terre mère et l'habitant qui la remue ! Et salut amical à Marcelin Albert, le grand agitateur du brave peuple de la terre ! De tout cœur.» Et finalement lors de la grande manifestation du 9 juin 1907, Mistral, d'un télégramme évasif : «Les patriotes de Provence, réunis aujourd'hui en Avignon aux fêtes du Palais des Papes, envoient à leurs compatriotes, les vignerons du Languedoc, le réconfort chaleureux de leur frater-

3. Robert LAFONT, Mistral ou l'illusion, Energas, Vent terral, 1980 [Paris, Gallimard, 1954], p. 341.

4. Les pancartes revendicatives furent souvent conservées «Aver tan de bon vin e pas poder manjar de pan», "Lo darnièr crostet» [Avoir tant de bon vin et pas pouvoir manger de pain], [Le dernier morceau de pain].

5. Jean-Pierre ChAmbon et Anne-Marguerite FrYBA-Reber, «Le Félibrige et le mouvement des vignerons de 1907 : quatre lettres inédites de Devoluy à Ronjat», Lengas, 1995, n 38, p. 10. 
nité et de leur pleine communion dans leurs revendications plus que justifiées ${ }^{6} . »$

Un paroxysme de cette mobilisation fut atteint avec la mutinerie du $17^{\mathrm{e}}$ de ligne. Au soir du 20 juin, à Agde, près de 600 troupiers ont levé la crosse en l'air et saisi des cartouches, puis se sont rendus à Béziers dont on les avait écartés la veille pour y installer des régiments plus «sûrs». Accueillis avec ferveur par la foule biterroise, ces vignerons sous l'uniforme ont forcé les barrages qui avaient été mis en place sans qu'aucune goutte de sang n'ait par eux été versée. Leur reddition au soir du 21 juin, puis leur exil forcé à Gafsa pour plusieurs mois ont clos l'épisode.

Revenant sur l'événement et le rapprochant du rêve fracassé lors de la bataille de Muret de 1213, s'exprimant lors du septième centenaire en 1913, Ferroul regretta que «l'Occitanie, la patrie occitane » ait été empêchée de constituer " une admirable nation ", et dans sa réflexion sur Mistral en 1954, Robert Lafont de déplorer « quelle belle chance négligée ${ }^{7}$ ! .

\section{La réplique de 1953}

Les mesures mises en œuvre pour réguler le marché du vin ne devaient pas empêcher le renouvellement des phénomènes de surproduction. Durant l'été 1953, la situation des viticulteurs des départements languedociens fut à nouveau très difficile: l'impossibilité d'écouler la récolte de 1952 les mettait en difficulté à l'approche des vendanges aussi la réactivation d'un comité régional de salut viticole débouchait sur des mesures inédites : l'édification de barrages routiers renouvelant la tradition des barricades dans un pays où l'automobile était en train de s'imposer. Comme le souvenir de 1907 était particulièrement vivant, les pancartes en occitan vont refleurir et la langue va être utilisée mettant notamment à profit le jeu de mots sur le surnom de Clémenceau (le tigre) et le nom du président du conseil Joseph Laniel (anelh : « agneau » en occitan).

6. Correspondance de Frédéric Mistral et E., F. et M. Jouveau (1877-1913), Aix-enProvence, s.n., 1993, p. 46.

7. Robert LAFONT, op. cit., p. 342. 


\section{La Sala - Decazeville}

Cette jonction entre les préoccupations de la langue et les préoccupations économiques et politiques devait être réalisée huit ans plus tard avec la lutte des mineurs de Decazeville. La grande grève de 1961-1962 est la plus longue de l'histoire du monde minier : soixante-six jours avec occupation des puits, doublée de grèves de la faim. Elle faisait suite à l'annonce brutale d'un plan de deux mille licenciements lancé par le pouvoir d'alors, plan élaboré par la Communauté européenne du charbon et de l'acier (CECA) qui prévoyait la fermeture de toutes les mines de Decazeville, pour rentabilité insuffisante. Le cadre est emblématique de la situation occitane : une entreprise nationale (Les charbonnages de France) décide de fermer un bassin sans avoir prévu quelque plan de reconversion que ce soit. Comme en 1907, c'est toute une population qui se mobilise, elle est consciente qu'elle ne maîtrise plus son destin et cherche dans la mobilisation régionale à se faire entendre du pouvoir central. La grève de Decazeville est porteuse de nouvelles formes de lutte ou de nouveaux thèmes : «Les idées de décentralisation, de mise en place de véritables structures régionales, de lutte contre les déséquilibres régionaux font leur chemin ${ }^{8}$. »

Serge Mallet publia dans l'hebdomadaire France Observateur, du 11 janvier 1962, un reportage intitulé : «la révolte des colonisés de l'intérieur». Synthétisant son propos, il s'exclamait « il s'agit de briser la structure coloniale, je dis bien coloniale, de la moitié des départements français ». Il estimait que l'union des paysans, des instituteurs, des cheminots autour de la lutte des mineurs posait un problème grave impossible à pallier par des mesures partielles et de conclure :

«Seuls de grands plans régionaux d'équipement, conçus à la fois autour de la création de pôles de développement et de l'utilisation rationnelle des productions primaires, c'est-à-dire en premier lieu de création sur place d'industries de transformation des produits agricoles génératrices d'autres activités industrielles, l'ouverture de grands axes ferroviaires et routiers à débit rapide à l'intérieur du fameux "désert français", le développement de

8. Philippe MARCY, «Une grève peu ordinaire : Decazeville 1961-1962 », Arkheia,» 2008. 
l'équipement scolaire et universitaire pour former sur place des techniciens et des ouvriers qualifiés modernes sont en mesure de bloquer la détérioration permanente, chaque jour aggravée, de la situation de ces départements de l'Ouest, du Centre, du SudOuest et du Midi. »

Mais Serge Mallet lançait un avertissement :

«Pour renverser le courant amorcé depuis les débuts du capitalisme industriel, il faut aussi s'attaquer au centralisme politique ; la décentralisation des pôles de développement économique ne se fera que parallèlement à la décentralisation des centres de direction politique. De Richelieu à De Gaulle, de Robespierre à Mollet et Thorez, il existe une constante de la concentration du pouvoir qui est véritablement une solide tradition nationale. C'est cet archaïsme qui fait de nos provinces un objet de musée. »

Pour les occitanistes, la grève de Decazeville provoqua la création du Comité occitan d'études et d'action (COEA) au sein duquel le déclin de la langue, le déclin économique et l'absence d'autonomie politique allaient être analysés dans le cadre du colonialisme intérieur.

Naturellement, de 1954 à 1962, la décolonisation était au cœur des préoccupations et François Fontan, en 1959, fonde le Parti nationaliste occitan et prône une stratégie de libération nationale qu'il appuiera sur une théorie de la politique, l'ethnisme'.

Mais plus que ces tentatives marginales, le constat du sousdéveloppement d'un «certain nombre de régions du territoire exmétropolitain » et des traits morbides qu'y présentent l'économie et la vie sociale est de plus en plus partagé dans les années 1960, et aux Rencontres socialistes de Grenoble de 1966, Michel Rocard présente un rapport intitulé Décoloniser la Province. L'écart est considérable entre les différentes composantes, les analyses de géographes ou d'historiens sur le sous-développement (Raymond Dugrand, André Armengaud), le désespoir de catégories sociales malmenées par le développement capitaliste, le mimétisme des situations coloniales,

9. François FONTAN, Ethnisme : vers un nationalisme humaniste, Bagnols-surCèze, Librairie Occitane, 1975. 
les remèdes technocratiques et la grande réussite de Robert Lafont sera de parvenir à agréger tous les éléments disparates dans la grande perspective de la Révolution régionaliste où il conclut que «le sous-développement ne peut être compris en France que selon des processus destructeurs liés à l'État, à l'anarchie du développement capitaliste que le centralisme protège ou laisse faire ${ }^{10} »$. Mais surtout il dégage le fonctionnement du sous-développement des régions françaises. À la base, il y a, concède-t-il, une situation d'infériorité des ressources peu susceptibles de soutenir une industrie moderne ${ }^{11}$. Mais cette infériorité devient un handicap dirimant dès lors que la région ne peut concevoir une protection et une animation de son industrialisation dans un contexte où les frais de transport constatés sur la base de réseaux qui n'ont pas été conçus pour son développement jouent contre elle. Ces deux mécanismes dévoilent le facteur essentiel, «l'absence catastrophique d'une vue de l'intérêt régional».

Cette base lui permet alors de rassembler un certain nombre de processus économiques dont le sous-développement régional est l'enveloppe perceptible dans le concept de «colonialisme intérieur». Il énumère ainsi :

1) la dépossession industrielle et l'investissement colonisateur,

2) la primauté des industries extractives sur les industries de transformation,

3) la dépossession du domaine agricole,

4) la dépossession des circuits de distribution,

5) la dépossession des ressources touristiques.

Finalement, il y a « un mécanisme identique, qu'on parle culture et langue ou qu'on parle économie ${ }^{12}$ » et le salut peut venir de l'économie si elle accouche d'une révolution sociale. « La révolution sociale est possible en France, elle correspond même au génie français le plus authentique. Mais il faut pour cela déterminer le grand point de rupture dans la société française actuelle : les problèmes économiques régionaux ${ }^{13}$. »

10. Robert LAFOnT, La Révolution régionaliste, Paris, Gallimard, 1967, p. 130.

11. Ibid., p. 131-132.

12. Ibid., p. 205.

13. Ibid., p.182. 


\section{La diffusion du concept de colonialisme intérieur}

Cette relation entre langues et difficultés économiques régionales va être déclinée dans diverses versions dans les années 1970, la plus empreinte de ce qu'était l'idéologie tiers-mondiste étant sans nul doute celle du Petit livre de l'Occitanie tandis qu'une version savante sous-tend l'Histoire d'Occitanie ${ }^{14}$ mais dans tous les cas, le salut de la langue apparaît dès lors lié au développement d'un « régionalisme révolutionnaire ${ }^{15} »$.

En même temps, la sociolinguistique est convoquée pour associer langue et économie. Dans le $\mathrm{n}^{\circ} 1 \mathrm{~d}^{\prime}$ Occitanie passé et présent, la revue de «Lutte occitane», Y. Couderc montre l'interdépendance entre le statut des langues dans la situation de diglossie et les autres aspects de la situation politico-économique car «les problèmes linguistiques ne sont pas et, de loin simplement, linguistiques » de sorte que la « disparition de la diglossie ne peut venir que de la disparition de la situation politico-économique qui l'a fait naître ${ }^{16} \gg$.

Une certaine cohérence entre langue et situation politicoéconomique est fournie par l'importance de la population agricole en Occitanie qui fournit la majorité des locuteurs de l'occitan et dans Décoloniser en France ${ }^{17}$, Robert Lafont reconnaît le rôle de la paysannerie pauvre dans le renversement de la situation politique, économique et linguistique qu'il appelle de ses vœux :

« Si la révolution est conçue surtout ouvrière à Paris, Lyon, Lille, comme à Turin ou Milan, par contre en Occitanie comme en Bretagne, comme en Italie du centre et du sud, elle doit faire une large part de responsabilités à la paysannerie, et à la paysannerie pauvre et angoissée. L'angoisse peut être une préface à la conscience $^{18}$. »

Il est facile de reconnaître dans ces argumentations une inspiration marxiste établissant une relation entre les infrastructures (les

14. Robert LAfont et André Armengaud (dir.), Histoire d'Occitanie, Paris, Hachette, 1979.

15. Robert LAFONT, La Révolution régionaliste, op. cit., p. 248.

16. Occitanie passé et présent, $\mathrm{n}^{\circ} 1$, p. 21.

17. Robert LAFOnT, Décoloniser en France, Paris, Gallimard, 1971.

18. Ibid., p. 295. 
conditions économiques) et les superstructures (culture et langues), relation dont on sait qu'elle ait été âprement débattue au tournant des années 1960 et 1970 autour d'Althusser et de la redécouverte de Gramsci. Mais comme Schumpeter l'a montré en raisonnant d'après et contre Marx à travers son idée de la création destructrice, le propre de l'économie capitaliste est de constamment rebattre les cartes et au moment où le déclin économique des régions méridionales était censé apporter le déclic révolutionnaire, commençait à se faire sentir la $«$ revanche du Sud $»^{19}$.

\section{Les stratégies linguistiques s'appuyant sur les ressorts économiques}

Les régions occitanes qui avaient connu un déclin relatif certain du XIX ${ }^{\mathrm{e}}$ siècle aux années 1970 voyaient au contraire leur situation s'améliorer par rapport aux régions qui avaient mieux réussi jusqu'aux «Trente Glorieuses» parce qu'elles étaient peu impactées par le déclin de la sidérurgie et l'essoufflement de l'industrie automobile qui y avaient été peu présentes. Au contraire, l'investissement dans l'éducation qui avait été la réponse des ménages au sous-emploi permettait une reconversion dans les industries de l'économie de la connaissance, tandis que l'industrie aéronautique dont la localisation était largement due à l'éloignement fortuit des frontières allemandes se développait avec l'aventure d'Airbus. Il y a donc une série de causes qui expliquent que des stratégies de reconquête linguistique faisant appel à des ressorts économiques aient fait leur apparition :

- un épuisement de l'espérance révolutionnaire et des stratégies prônant les ruptures radicales,

- une tendance à l'individualisation des stratégies professionnelles,

- une réaction vis-à-vis de l'uniformisation des produits et des modes de vie de sorte qu'on voit apparaitre un marketing de la provenance.

19. A. Berger, J. Catanzano, J.-D. Fornairon, J. Rouzier, La revanche du Sud. Un défi à la France, Paris, L'Harmattan, Logiques Sociales, 1988 ; René UHRICH, La France inverse : les Régions en mutation, Paris, Economica, 1987. 
Cette situation n'est pas spécifique à l'Occitanie. Ainsi après le rétablissement de la Generalitat en 1977, la politique linguistique catalane s'était fixé comme objectifs d'abord le rétablissement du catalan dans l'administration, puis dans le domaine de l'éducation et enfin dans les moyens de communications de masse (notamment via la création d'une station de télévision catalane - TV3). Parmi les objectifs restant pour aboutir à une «normalisation » de la langue restait l'usage dans le monde économique ${ }^{20}$.

Pour cela des enquêtes d'opinion furent conduites pour déterminer l'attitude de la population habitant en Catalogne vis-à-vis de la langue dans les activités économiques. Elles démontraient que la population y verrait un souci de témoigner une attention particulière à la Catalogne et donc un facteur positif pour les entreprises qui feraient ce choix. Ainsi les préoccupations étaient doubles : d'une part, convaincre les entreprises liées à la Catalogne qu'elles n'avaient rien à perdre à utiliser le catalan et d'autre part, montrer que les entreprises en général pouvaient espérer améliorer leur image en Catalogne par l'utilisation du catalan.

Cette politique de la Generalitat était bien en phase avec la stratégie de Jordi Pujol, le grand catalaniste du $\mathrm{XX}^{\mathrm{e}}$ siècle qui, dès 1960, donna comme objectif au catalanisme de «fer pais : c'est-àdire de doter la Catalogne des éléments non strictement politiques dont a besoin un pays pour fonctionner».

\section{La CEEPOc et les labels linguistiques}

Les marques collectives peuvent faire appel plus ou moins directement à l'attachement au territoire et/ou à la langue spécifique pour gagner un avantage concurrentiel, un attachement régulièrement attesté par les enquêtes sur l'occitan, attachement qui peut être le fait des habitants des régions de langue d'oc mais aussi à l'extérieur. Cette utilisation de la référence linguistique se manifeste dans les raisons sociales et intitulés des produits et des marques. Il n'apparaît pas que cette référence soit forcément synonyme d'attachement à la langue. Le paroxysme de la tension entre promotion de la langue et

20. Pere Duran Farell et Àngel Hevia i Carreras (dir.), La llengua en el món del comerç. : l'actitud dels catalans davant l'ús comercial del català, Barcelona, Direcció General de Política Lingüística, 1988. 
promotion de produits marqués linguistiquement est atteint dans le cas de «L'Occitane en Provence» ${ }^{21}$. À l'opposé, c'est pour appuyer une stratégie de reconquête linguistique que se sont mis en place des groupements de producteurs clairement basés sur la langue. La cohabitation entre cet objectif de promotion d'une langue et le calcul économique consistant pour des locuteurs à tirer profit de leur usage de la langue n'est pas facile comme les expériences depuis deux décennies le montrent.

Créée en 1993, la chambre économique européenne des pays Occitans (CEEPOc) entendait offrir aux entreprises qui en éprouvent le besoin des occasions d'échanges et de travail pour améliorer leurs résultats. Elle était « convaincue que la dynamique des entreprises est d'abord celle de leurs milieux; et que leur bonne santé économique n'est pas sans rapport avec les spécificités des territoires où elles se redéployent». Elle se donnait quatre missions :

- de représenter les acteurs économiques d'un territoire,

- de construire une économie en l'ouvrant sur le monde,

- d'accompagner les associations et les entreprises dans leur développement,

- de moderniser l'usage de l'occitan.

Les trois premières missions sont classique pour des associations professionnelles et l'accompagnement proposé essentiellement aux «petites structures» prenait place dans un créneau déjà largement labouré par d'autres initiatives (boutiques de gestion, structures d'aide au développement) vis-à-vis duquel la CEEPOc n'avait d'autre avantage comparatif que l'hypothétique relation culture/économie. C'est pourquoi la quatrième mission de la CEEPOc insistait sur cette spécificité et voulait «moderniser l'usage de l'occitan». Refusant de voir dans la langue occitane «une simple relique du passé ou un seul témoignage artistique», la CEEPOc voulait lui «permettre d'entrer dans la modernité en traduisant les mots de l'entreprise et de l'économie ». Elle propose à ses membres et à ses partenaires un lexique occitan des termes comptables, économiques, juridiques et

21. L'utilisation des références au territoire et/ou à la langue et son évolution mériteraient des études particulières. 
financiers de notre temps, traduit les documents qui lui sont présentés et ainsi encourage la diffusion en langue occitane des services et des produits de ce territoire.

En 2003, son promoteur René Pons dressait un bilan de ses activités ${ }^{22}$ qui concernaient d'une part la création et la gestion d'événements autour de produits occitans et d'autre part le lancement de deux labels.

La CEEPOc a été à l'origine ou la cheville ouvrière de nombreux «événements» qui ont sans nul doute accru sa notoriété dans les milieux économiques.

- Les Mercat occitan [les marchés occitans] : il s'agit d'organiser, au travers d'un véritable marché centré sur les produits du terroir une animation à caractère fortement occitan ce qui correspond aux objectifs de la CEEPOc, à savoir l'animation économique à partir du caractère culturel occitan (villages et petites villes).

- Les Fièiras d'Occitània [les foires d'Occitanie]. Vitrines des produits occitans : culturels, agroalimentaires, artisanats, tourisme, industrie, sports, environnement et cadre de vie, gastronomie (Montpellier, Nîmes).

- Les Auberga occitana [les auberges occitanes] : réseau de professionnels désireux de développer la promotion de la gastronomie traditionnelle dans une ambiance occitane.

- Les Collòquis [les colloques] : colloques thématiques : tourisme et architecture.

À coté de ces événements, l'activité essentielle de la CEEPOc a tourné autour de labels de qualité et d'identité qu'il est tout à fait intéressant d'étudier. La CEEPOc avait réfléchi au positionnement des produits labellisés tout particulièrement pour les produits agroalimentaires pour lesquels elle avait élaboré une véritable charte de qualité comportant les éléments suivants :

- Identité culturelle : la prise en compte de celle-ci se concrétise par la mise en avant de la culture et de la langue du terroir (ici langue

22. René PONS, «15 minutes pour convaincre », synopsis d'une intervention publique. 
d'oc) au niveau de la communication et de l'étiquette. Cet élément est décisif pour réaliser la démarche identitaire.

- Authenticité.

- Qualité.

- Patrimoine/Racines : la valorisation conjointe de la culture, de la langue et du savoir-faire constitue une défense du patrimoine régional mais également une mise en valeur et une évolution de ce patrimoine vers des tendances de consommation évolutives. En outre, ce critère valorise les souvenirs enfouis mais encore puissants des expériences passées et lointaines.

- Terroir : ce critère se caractérise par une mise en valeur du pays et de tous les symboles qui y sont associés. À noter que la langue occitane est restée dans l'imaginaire collectif très attachée au paysage et à la nature.

- Originalité : les produits ou services associés à cette démarche se caractérisent par leur nouveauté et leur différenciation accentuée par une mise en avant forte de l'identité culturelle et la valorisation de produits peu pris en compte par les distributeurs.

- Goûts.

- Fabrication artisanale : celle-ci va généralement de pair avec les critères exprimés précédemment compte tenu des cibles à atteindre (vente locale, distribution en ville, exportation dans des régions sensibilisées à l'identité).

Dans ce cadre, la CEEPOc a créé et déposé le label Produch occitan et initie un réseau de qualité Auberga occitana avec le secteur de la restauration.

À défaut d'une étude détaillée des caractéristiques des adhérents, il est difficile de positionner précisément le label Produch occitan entre les trois sommets du triangle culture - langue - économie. Sous réserve de cet inventaire, on relèvera qu'il s'agit essentiellement de promouvoir des produits et que la langue n'est qu'une référence vague parmi d'autres.

Le réseau de qualité Auberga occitana était plus élaboré et faisait référence de façon explicite au marqueur linguistique. Il se présente comme un réseau de promotion de la gastronomie traditionnelle dans un environnement occitan. Il s'agit donc pour des professionnels de mettre en commun des efforts de promotion vis-à-vis de la clientèle 
en offrant des prestations répondant à certains standards de qualité qui concernent aussi bien l'usage de la langue que les autres éléments de la restauration.

\section{Leçons de l'expérience CEEPOc}

Le fonctionnement de la CEEPOc reposait quasi exclusivement sur le bénévolat et l'appel aux bonnes volontés. Alors qu'elle tentait de rassembler des actions dans six régions, les aides reçues au niveau national était d'un montant ridicule : ainsi nous avons pu trouver des documents du Sénat fournissant la «liste des associations régies par la loi du $1^{\text {er }}$ juillet 1901 ayant reçu directement sur le plan national une subvention à quelque titre que ce soit» en 1999/2000 et 2003/2004. La CEEPOc figure une seule année (2003) pour un montant de 4000 euros. À titre de comparaison, les associations loi de 1901 promouvant le jeu de boules provençal empochaient chaque année 250000 euros $^{23}$. Avec ses moyens, elle a réussi à créer des événements sans que les retombées, en termes de retombées pour elle-même, pour la langue ou pour l'économie régionale, n'aient été mesurées. En ce qui concerne les labels, en se positionnant sur les produits agroalimentaires, elle entrait en concurrence avec les labels de qualité au risque de brouiller son message. En mettant l'accent sur les petites structures et l'artisanat, elle espérait attirer des activités peu prises en compte dans les organisations consulaires officielles mais elle courait le risque de la marginalisation.

Alors que la dynamique de la CEEPOc s'essoufflait, apparaissait l'Agéncia de desvolopament de l'occitan. L'ADOC Tarn est une association loi 1901, créée en 2004, œuvrant dans les domaines de l'économie et du tourisme. Son objectif est de faire la preuve que la langue et la culture occitanes sont des atouts véritables pour la création et valorisation des produits. Cet objectif est intégré dans la conception des divers projets concernant le commerce, les services, la communication, les créations d'animations et de produits, les circuits et les animations touristiques. Un partenariat peut s'établir avec des associations, des institutions, des entreprises, des commerçants, artisans, des restaurants, syndicats professionnels, etc.

23. Cf. Le récapitulatif des subventions aux associations dans http://www4.minefi. gouv.fr/budget/plf2004/somjau04.htm, consulté le 26/12/2013. 
Tant la CEEPOc que l'ADOC mettaient en avant la promotion économique des producteurs qu'elles voulaient regrouper. Mais l'expérience a montré les tiraillements qui pouvaient apparaitre entre promotion de la langue et promotion des utilisateurs de la langue. Que ce soit pour les événements ou pour les besoins des labellisés, l'expérience de la CEEPOc fait apparaitre le besoin d'une relation étroite avec les spécialistes de la langue. Parmi les services proposés par la CEEPOc figuraient des documents de référence sur la langue et des lexiques élaborés par le GIDILOC. Ce «Groupe d'initiative pour un dictionnaire informatisé de la langue occitane» est une association loi de 1901. Sa responsable Josiane Ubaud ${ }^{24}$ se présente comme «professeur de mathématiques pendant huit ans, puis lexicographe en domaine occitan, formatrice en ethnobotanique à l'IUFM». Le GIDILOC est le correspondant occitan du projet Linmiter dans le cadre des actions de sauvegarde et de développement des langues régionales et/ou minoritaires menées au niveau européen. Il a été subventionné dès sa création par la Commission des Communautés européennes. La comparaison des objectifs et réalisations du GIDILOC fait apparaitre cruellement la situation difficile des «industries de la langue» dans le domaine occitan. Reposant sur des initiatives dispersées ${ }^{25}$, faisant appel tantôt au militantisme ou se raccrochant à des projets universitaires soumis à des plans de carrière individuels, la production en matière linguistique est incapable de suivre dans l'état actuel une tentative sérieuse de lier préoccupations économiques et linguistiques.

Les limites de cette convergence apparaissent clairement dans les relations entre la CEEPOc et le Servici de la lenga occitana ${ }^{26}$. Ce dernier était une émanation de la politique linguistique de la Région

24. http://www.futura-sciences.com/magazines/nature/infos/personnalites/d/ botanique-josiane-ubaud-54/, consulté le 28/10/2013.

25. Depuis plusieurs siècles, il ne manque pas d'érudits lexicologues ayant entrepris des dictionnaires de l'occitan. Citons au cours des dernières décennies, le Diccionari General Occitan de Joan de Cantalausa (premier dictionnaire, occitan-occitan). Mentionnons aussi la tentative de créer un dictionnaire en ligne fonctionnant aussi bien du français vers l'occitan que l'inverse sur http://www. panoccitan.org/diccionari.aspx.

26. Patrick SAUZET, «Vers un Service de la langue occitane en Languedoc-Roussillon», Dynamiques institutionnelles et patrimoines linguistiques de l'Aquitaine, Bordeaux, M.S.H.A., 1996, p. 133-144. 
Languedoc-Roussillon dont l'artisan avait été Étienne Hammel, chargé de mission pour les langues et cultures occitane et catalane à la Région Languedoc-Roussillon de 1985 à 1998. Basé à l'université Paul Valéry de Montpellier, le Servici visait à offrir des prestations en langue occitane. Jean-François Courouau, son premier animateur, réfléchissant sur cette expérience écrit :

«Mais il est une utilisation du symbole qui n'est pas toujours dénuée d'arrière-pensées économiques. Le Servici de la lenga occitana en a eu la preuve lors de l'organisation de la première grande foire des pays d'Oc baptisée Comédie d'Occitanie qui s'est déroulée sur la place de la comédie à Montpellier les 5 et 6 juin 1996. Ici aussi, le promoteur, René Pons, président de la CEEPOc, concevait la langue comme vecteur d'une symbolique et d'une dynamique. Les pays d'oc produisent et exportent des produits - de qualité, diront les producteurs - qui ont tout à gagner à jouer la carte de la caractérisation maximale. La langue occitane en ce qu'elle est éminemment liée à l'image du Sud est un élément essentiel pour valoriser l'origine géographique d'un produit de préférence lié au terroir (vin, miel, confitures, bonbons, biscuits, jambons, saucisses, etc.). Malheureusement, là encore, les mêmes blocages que chez certains décideurs politiques ou administratifs ont pu être observés et si certains producteurs ont présenté leurs produits grâce à un étiquetage monolingue occitan ou bilingue, d'autres ont montré la plus grande stupeur quand il leur a été demandé pourquoi il n'y avait pas une ligne d'occitan à leur stand. La réponse « les gens vont pas comprendre » permet de s'économiser la peine de faire appel à un traducteur pour les non-occitanophones mais surtout elle révèle le présupposé d'une inopérance sociale de l'occitan ${ }^{27}$. »

\section{L'Ofici per l'occitan et le label Òc per l'occitan}

Il s'agit là encore d'une association loi de 1901 dont la création est datée du 4 avril 2006. Le projet qui devait démarrer concrètement en 2007 se définit par son objet «repérer et faire connaître les

27. Jean-François Courouau, «La présence de l'occitan dans la vie publique : blocages et évolutions vus par le servici de la Lenga occitana », Dix siècles d'usages et d'images de l'occitan, des troubadours à l'internet, Henri Boyer et Philippe Gardy (dir.), Paris, l'Harmattan, 2001, p. 319-329. 
entreprises et organismes de toute sorte qui contribuent à faire vivre la langue occitane ; son action vise aussi à susciter le développement de nouveaux acteurs sociaux qui participeront à leur tour à ce mouvement de promotion linguistique». On peut noter qu'il est plus précis que les missions que s'était fixées la CEEPOc puisqu'est visée très clairement la promotion linguistique. Mais comme on le verra, il n'échappe pas à certaines ambigüités et il bute sur les mêmes difficultés que la CEEPOc.

Son activité a consisté principalement à promouvoir un label $O \mathrm{C}$ per l'occitan [«D'accord pour l'occitan»] destiné à identifier et à afficher les dispositions à l'égard de la langue occitane des adhérents au label. Trois degrés d'implication sont distingués. Le premier niveau («L'occitan nous plaît») consiste simplement à manifester de bonnes dispositions vis-à-vis de la langue et un engagement à mettre en œuvre quelques mesures pour valoriser, montrer ou faire entendre la langue. Sont énumérées des actions susceptibles d'être mises en œuvre : affichage de la langue écrite, signalétique au moins partielle, sur tout type de document public comme les menus, les catalogues, site internet, la publicité, les factures, l'étiquetage, l'enseigne, etc. mais aussi des animations et promotions intégrant la langue occitane. Sont également prônées la participation du personnel à des cours de langues ou l'adhésion à un club occitan de façon à assurer qu'une personne au moins en contact avec le public peut utiliser la langue et enregistrer des annonces orales bilingues.

Peuvent prétendre au niveau 2 (« Ici on parle occitan»), les entreprises qui offrent une possibilité de service en occitan. Selon la taille, une ou deux personnes au moins doivent être en mesure d'offrir leurs services en occitan. Le niveau 3 est réservé à celles des entreprises capables d'utiliser l'occitan comme langue de travail habituelle. La majorité du personnel a une maitrise de la langue orale et écrite. L'affichage donne la priorité à l'occitan tandis que la priorité est donnée dans les échanges aux entreprises. Le même effort se retrouve dans la communication sur les produits, les clients, les fournisseurs. Le niveau 3 correspond à une réappropriation totale de la langue.

Le nom de l'association Ofici per l'occitan était clairement une référence aux besoins d'une production terminologique qui semble bien être le déclic pour engager une dynamique langue - économie 
et le fonctionnement de l'Ofici a montré que sans l'appui d'une structure susceptible de fournir des services linguistiques le projet ne pourrait fonctionner.

L'Ofici a démarré par l'engagement personnel d'une personne qui a accepté de renoncer à des activités économiques rémunératrices (un restaurant occitan) pour se lancer dans le projet à mi-temps. Aucun investissement en matière de communication ne pouvait être réalisé et l'Ofici n'a bénéficié en matière de médias que d'une présentation au magazine occitan de FR3 Midi-Pyrénées, de deux petits articles dans le journal local La Dépêche et de mentions dans la presse occitane. Au bout de deux ans, un nouveau chargé de recrutement a été embauché pour le Tarn qui apparaissait comme une cible privilégiée en raison du rôle témoin qu'il est censé jouer dans la politique linguistique de la Région Midi-Pyrénées. Au départ, l'autonomie financière était souhaitée même si un complément de financement initial paraissait souhaitable. Mais très vite l'autonomie financière est apparue inaccessible et le recours à un financement public indispensable de sorte que l'imbrication avec l'institut d'études occitanes s'est renforcée à travers des conventions entre les deux associations.

En contrepartie de l'adhésion, l'Ofici fournit un annuaire papier annuel dont le tirage s'est élevé jusqu'à 100000 exemplaires, présent dans de nombreux offices du tourisme et chez les labellisés euxmêmes. Une première a été élaborée par un membre bénévole de l'Ofici puis une seconde version a fait l'objet d'un contrat avec un professionnel destiné à rendre le design du site moins austère mais là encore l'investissement en terme de site est très loin des niveaux atteints par les fournisseurs de sites professionnels. Surtout que, pour un site à vocation linguistique, il ne propose pas de faire entendre la langue.

\section{Réflexions sur les labels linguistiques}

\section{Compétences linguistiques et labellisés}

L'expérience du label comme celle de la CEEPOc montre qu'il existe des responsables d'entreprises qui souhaitent utiliser la langue pour leur communication commerciale mais les statuts 
de l'Ofici sont ambigus vis-à-vis de la place des labellisés dans le pilotage du label. L'Ofici joue le rôle d'un organisme certificateur des compétences linguistiques de sorte que le rôle des labellisés dans le fonctionnement et l'orientation de l'association apparaît flou. Il n'est pas facile d'articuler un fonctionnement démocratique dans lequel les labellisés piloteraient l'évolution du label avec une fonction d'évaluation. En outre, on peut se demander si les niveaux tels qu'ils ont été imaginés au départ ne sont pas inadaptés aux pratiques et compétences langagières réelles.

\section{Label et qualité des produits et des services}

De façon inévitable, les labels se sont répandus surtout dans les activités liées au territoire (agroalimentaire, hôtellerie-restauration, tourisme). La compétence linguistique est incontestablement une dimension de la qualité des biens et services de ces entreprises et la relation au territoire entre dans la perspective des locavores, à la recherche d'authenticité, etc. Mais vis-à-vis de ces dimensions, il est clair que le label linguistique rentre en concurrence avec d'autres tentatives de mettre en avant la provenance géographique. Cette concurrence est sensible au niveau des efforts des collectivités territoriales dont le soutien à des labels « Produit en Bretagne» ou « Produits du Sud-Ouest » sont sans commune mesure avec leur soutien au label linguistique. Plus généralement pour un consommateur y a beaucoup d'autres dimensions de sorte que le positionnement du label Oc per l'occitan par rapport aux autres labels de qualité devrait être précisé.

\section{Notoriété et effets de seuils}

Au bout de sept ans, il apparait clairement que le label ne peut pas dans les conditions actuelles atteindre un équilibre économique. La prospection par un commercial est extrêmement chronophage et les résultats à l'heure actuelle ne permettent pas une dynamique autoentretenue. L'affichage du label, le site internet et l'annuaire permettent certes un retour pour les entreprises même s'il n'est pas mesuré mais il n'est sans doute pas suffisant pour entrainer par lui même une dynamique propre. 
On peut se demander sur la base de l'expérience de la CEEPOc et à présent avec le recul dont on dispose pour évaluer le label, si les moyens mis en œuvre sont suffisants pour assurer la notoriété qui permettrait d'enclencher un cercle vertueux. Les comparaisons avec des marques collectives comme «Pavillon France ${ }^{28}$ » sont à cet égard édifiantes. Le lancement en 2012 de la marque collective a mobilisé $5 \mathrm{M}$ d'euros. Concernant la marque «Produit du SudOuest de France », les régions Midi-Pyrénées et Aquitaine y ont consacré chacune 500000 euros en 2012 et le même montant en 2013. Ces chiffres relativisent le budget annuel moyen du label (hors prestations militantes) qui n'a jamais dépassé les 20000 euros absorbés essentiellement par la logistique du label de sorte que les dépenses de communication ont été très réduites.

\section{Conclusion}

L'Ofici paraît dans une position inconfortable : il existe certainement une demande de services linguistiques de la part d'acteurs économiques (traductions, signalétique, communication, etc.) mais l'Ofici avec ses moyens ne peut fournir ces services qui correspondraient à ceux d'un véritable office de la langue. Le cercle vertueux espéré n'a jusqu'à présent pas été vraiment au rendez vous.

Finalement, on a envie de revenir vers un grand stratège et dire «il faut compter sur ses propres forces». Ce qui peut s'entendre dans différents sens. D'abord, les sondages montrent régulièrement l'aura positive dont bénéficie la langue d'oc, il conviendrait d'étudier davantage les contours de cette image pour améliorer la communication du label qui ne doit pas se contenter de l'écrit (papier ou html). Ensuite, on connaît les problèmes de graphies et l'émiettement des militants linguistiques, il importe donc que le label soit clairement ouvert à tous ceux qui ont le souci de la relation langueéconomie. Enfin, un label qui fait appel aux professionnels doit leur faire toute leur place dans le pilotage et l'orientation.

\section{$*$}

28. http://www.pavillonfrance.fr/. 
L'auteur remercie René Pons, Patric Sauzet, Jean-François Courouau pour leur aide, les participants au congrès Cultures régionales et developpement économique des 30 et 31 mai 2013 (Marseille Luminy) et au séminaire de la Bretagne linguistique du 15 juin 2013 pour leurs remarques et suggestions. Il reste seul responsable des erreurs qui peuvent subsister. 